\section{THU0045 IL-25/IL-17RB AXIS IS ACTIVATED AND ASSOCIATED WITH ILC2 EXPANSION IN GRANULOMATOSIS WITH POLYANGIITIS (GPA)}

Daniele Mauro $^{1,2}$, Giuliana Guggino $^{1}$, Angelo Ferrante ${ }^{1}$, Cristiano Alessandri ${ }^{3}$, Serena Fasano ${ }^{4}$, Daniela lacono ${ }^{4}$, Marianna Lo Pizzo ${ }^{5}$, Riccardo Alessandro ${ }^{5}$, Aroldo Rizzo ${ }^{6}$, Francesco Ciccia ${ }^{4} .{ }^{1}$ University of Palermo, Dipartimento biomedico di Medicina Interna e Specialistica - Sezione di Reumatologia, Palermo, Italy; ${ }^{2}$ Queen Mary University of London - William Harvey Research Institute, Experimental Medicine and Rheumatology, London, United Kingdom; ${ }^{3}$ Sapienza University of Rome, Department of Internal Medicine, Rome, Italy; ${ }^{4}$ University of Campania "Luigi Vanvitelli", Rheumatology Section, Naples, Italy; ${ }^{5}$ University of Palermo, Dipartimento di Biopatologia e Biotecnologie Mediche e Forensi, Palermo, Italy; ${ }^{6}$ Azienda Ospedaliera Ospedali riuniti Villa Sofia-Cervello, Pathology Unit, Palermo, Italy

Background: Pathogenesis of Granulomatosis with polyangiitis (GPA) is still unknown. However, it has been observed a skewing of circulating CD4+ T cells toward the Th17 and Th2 phenotype. The pro-inflammatory cytokine interleukin 25 (IL-25) is a member of IL-17 cytokine family associated to the Th2 immune phenotype. Through the receptor IL17RB, IL-25 further sustains the Th2-type immune response and elicits the expansion of the type 2 innate lymphoid cells (ILC2) and M2 macrophages. A pathogenic role of the innate lymphoid cells in GPA has been recently demonstrated; however, the relevance of IL-25 in this condition remains unexplored.

Objectives: Aim of the study was to evaluate the expression of IL-25/IL$25 \mathrm{R}$ axis and its functional relevance in patients with granulomatosis with polyangiitis (GPA).

Methods: Thirty patients with a diagnosis of GPA fulfilling the Chapel Hill Consensus Conference and ACR classification criteria, and 20 agematched healthy controls $(\mathrm{HC})$ were included in this study: age range 30-66 years, 40\% female in GPA group and $32-63$ years, $20 \%$ female in HC. IL-25 and IL-33 serum levels were measured by ELISA at the diagnosis and after Rituximab (RTX) therapy $(n=15)$. Immunohistochemical staining for IL-25, IL-33, IL-17B was performed on sections of renal biopsies obtained from GPA patients $(n=10)$. Kidney ILC2 infiltration was assessed by confocal microscopy. Frequencies ILC1, ILC2 and ILC3 were evaluated by flow cytometry analysis by CD45, Lyn, CRTH2, GATA3, Tbet, CD56, NKp44, IL-22, IL-23R, RORc. Expression levels of IL-5 and IL-13 in kidney were quantified by quantitative real-time PCR (qRT-PCR). Results: Increased pre-RTX serum levels of IL-25 and IL-33 at baseline were observed in GPA compared to HC $(p<0.0001)$. Both serum IL-25 and IL-33 correlated with the Birmingham Vasculitis Activity Score (BVAS) $\left(r^{2} 0.51 p<0.0001\right.$ and $r^{2} 0.14 p<0.05$ respectively). IL-25, IL-17RB and IL33 expressing cells were increased in GPA renal biopsies vs $\mathrm{HC}$ $(p<0.0001)$. Frequencies of circulating ILC1 and ILC3 were not significantly increased in GPA. Conversely, the frequency of ILC2 was expanded in GPA vs HC $(p<0.0001)$ and correlated with BVAS $\left(r^{2} 0.60\right.$ $\mathrm{p}<0.0001)$. The number of ILC2 and the levels of expression of IL- 5 and IL-13 in GPA kidney were increased $(p<0.0001)$. RTX therapy led to a reduction in IL-25 and IL-33 serum levels and in the frequency of circulating ILC2.

Conclusion: Our data confirm the increases in IL-33 levels previously reported by other authors. The increased circulating and tissue levels of IL-25 and IL-25 receptor-expressing cells, including ILC2, suggests a role for the IL-25/L-17RB axis in GPA pathogenesis. Treatment with antiCD20 Rituximab may lead to modulation of IL-25/IL-17RB axis and reduction in ILC2.

Disclosure of Interests: Daniele Mauro: None declared, Giuliana Guggino Grant/research support from: Laborest, Pfizer, Consultant for: Novartis, Abbvie, Speakers bureau: Sandoz, Angelo Ferrante: None declared, cristiano alessandri: None declared, SERENA FASANO: None declared, Daniela lacono Speakers bureau: PFIZER, Marianna Lo Pizzo: None declared, Riccardo Alessandro: None declared, Aroldo Rizzo: None declared, Francesco Ciccia Grant/research support from: CELGENE, PFIZER, Consultant for: UCB, NOVARTIS, CELGENE, PFIZER, LILLY, Paid instructor for: UCB, NOVARTIS, CELGENE, PFIZER, LILLY, JANSSEN, Speakers bureau: UCB, NOVARTIS, CELGENE, PFIZER, LILLY, JANSSEN, ROCHE, AMGEN

DOI: 10.1136/annrheumdis-2019-eular.4072

\section{THU0046 \\ FRACTALKINE PROMOTES DIFFERENTIATION INTO OSTEOCLASTS FROM HUMAN PERIPHERAL BLOOD MONOCYTES}

Sei Muraoka ${ }^{1}$, Kaichi Kaneko ${ }^{1}$, Natsuko Kusunoki ${ }^{1}{ }^{1,2}$, Shinichi Kawai ${ }^{2}$, Toshihiro Nanki ${ }^{1} .{ }^{1}$ Toho University, Division of Rheumatology, Department of International Medicine, Tokyo, Japan; ${ }^{2}$ Toho University, Department of Inflammation and Pain Control Research, Tokyo, Japan

Background: Fractalkine (FKN) also known as chemokine (C-X3-C motif) ligand 1 plays important roles in the pathogenesis of rheumatoid arthritis (RA). Clinical trials of monoclonal antibody against FKN for RA are currently in progress. Although FKN has been reported to be involved in the migration of osteoclast precursor cells, the direct effect of FKN on osteoclast differentiation is not clear.

Objectives: In this study, we aimed to clarify the effect of FKN on differentiation into osteoclasts from human peripheral blood monocytes.

Methods: CD14-positive monocytes were purified from peripheral blood obtained from healthy donors by magnetic beads. CD16-positive and CD16-negative peripheral blood monocytes were isolated from the CD14positive monocytes. The purified peripheral blood monocytes were incubated with M-CSF + RANKL with or without FKN for 1 week. The cells were stained for tartrate-resistant acid phosphatase (TRAP). TRAP-positive multinucleated (three or more nuclei) cells were counted as osteoclasts The cells were seeded onto plates coated with calcium phosphate thin films, and the resorption lacunae were examined. Next, CD14-positive monocytes were incubated with GM-CSF + IL-4 with or without FKN for 1 week to differentiate into dendritic cells. The surface markers of these cells were analyzed by flow cytometry. In addition, the monocyte-derived dendritic cells were further incubated with M-CSF + RANKL with or without FKN. Then the cells were stained for TRAP. The research procedure has been approved by the ethics committee of Toho University School of Medicine (Approval number: 27060).

Results: CD16-negative peripheral blood monocytes differentiated into osteoclasts by incubation with M-CSF + RANKL. The osteoclast differentiation was enhanced by additional stimulation with FKN in a concentration-dependent manner. In addition, calcium resorption was also enhanced by addition of FKN. On the other hand, CD16-positive monocytes treated with M-CSF + RANKL did not differentiate into osteoclasts with or without FKN. CD14-positive monocytes differentiated into dendritic cells by stimulation with GM-CSF + IL-4. Expression of CD14 was decreased and CD1a expression was increased on the monocyte-derived dendritic cells. CD16-negative monocyte-derived dendritic cells differentiated into osteoclasts by further stimulation with M-CSF + RANKL. By co-stimulation with FKN, the osteoclast differentiation from CD16-negative monocyte-derived dendritic cells was also enhanced.

Conclusion: FKN promotes osteoclast differentiation from human peripheral blood CD16-negative monocytes and also from CD16-negative monocyte-derived dendritic cells. FKN might contribute bone destruction in the affected joints of RA as well as inflammatory cell accumulation into the synovial tissue.

\section{REFERENCES}

[1] Tanaka Y, Takeuchi T, Umehara H, Nanki T, et al. Safety, pharmacokinetics, and efficacy of E6011, an antifractalkine monoclonal antibody, in a first-in-patient phase 1/2 study on rheumatoid arthritis. Mod Rheumatol. 2018; 28: 58

[2] Nanki T, Imai T, Kawai S. Fractalkine/CX3CL1 in rheumatoid arthritis. Mod Rheumatol. 2017; 27: 392.

[3] Nanki T, et al. Inhibition of fractalkine ameliorates murine collagen-induced arthritis. J Immunol. 2004; 173: 7010.

[4] Nanki T, et al. Migration of CX3CR1-positive T cells producing type 1 cytokines and cytotoxic molecules into the synovium of patients with rheumatoid arthritis. Arthritis Rheum. 2002; 46: 2878.

Disclosure of Interests: Sei Muraoka Grant/research support from: Novartis, Speakers bureau: Pfizer, Chugai, Kaichi Kaneko: None declared, Natsuko Kusunoki: None declared, Shinichi Kawai Grant/research support from: Nippon Zoki, Chugai, Ono, Ayumi, Hisamitu, Eisai, Japan Tobacco, Nippon Kayaku, Daiichi Sankyo, Astellas, Yutoku, and Mitsubishi Tanabe, Consultant for: Santen, Japan Tobacco, Speakers bureau: Ayumi, Chugai, Ono, Astellas, Pfizer, Celltrion, Toshihiro Nanki Grant/research support from: Chugai, Eisai, Takeda, Teijin, Eli Lilly, Bristol-Myers, AbbVie, Ono, Novartis, Asahikasei, Mitsubishi-Tanabe, Astellas, Ayumi, Pfizer, Daiich Sankyo, Shionogi, Sanofi, Nippon Kayaku, Yutoku, Actelion, UCB, Bayer, Nihon Pharmaceutical., Consultant for: UCB, Eisai, Chugai, Ono, Gilead. Speakers bureau: Mitsubishi-Tanabe, Chugai, Eisai, Takeda, Astellas, Janssen, Eli Lilly, Ayumi, Pfizer, Asahikasei, Sanofi, Daiichi Sankyo, Otsuka, AbbVie, Ono, Teijin, Nippon Kayaku, UCB. 